\title{
Performance ANd Interference Analysis of 802.11G WIRELESS NETWORK
}

\author{
Mingming $\mathrm{Li}^{1}$, Haiyang Liu ${ }^{1}$, Haifeng $\operatorname{Tan}^{2}$ and Miao Yang ${ }^{1}$ \\ ${ }^{1}$ State Radio Monitoring Center, Beijing and 100037, China \\ ${ }^{2}$ Beijing University of Posts and Telecommunications, Beijing and 100876, China
}

\begin{abstract}
This paper mainly presents Access Point s' (APs') performance and co-channel, adjacent channel interference according to $802.11 \mathrm{~g}$ standard. Firstly, our study illustrates the performance of one AP, including its coverage performance, load-carrying properties and fairness. Next we propose the details about co-channel, adjacent channel interference which should be paid attention to in deploying network services. Then, performance analyses are evaluated by simulation and real test for a dense wireless network. Our contribution is that the co-channel, adjacent channel interference analysis, the simulation and test results can be the basis offered to network operators
\end{abstract}

\section{KEYWORDS}

$802.11 g$ network, Coverage performance, Fairness, Throughput, Co-channel Interference, adjacent channel interference

\section{INTRODUCTION}

The WLAN network with IEEE $802.11 \mathrm{~g}$ standard is one most-widely deployed as its high speed and proven techniques. This technology operates in the $2.4 \mathrm{GHz}$ ISM (Industrial, Scientific, and Medical) radio spectrum with signal bandwidth 20MHz. IEEE Std. 802.11g-2003[1], part 11 gives the specifications about 802.11g's MAC layer and physical Layer (PHY). Extended Rate PHY is the proprietary vocabulary for $802.11 \mathrm{~g}$ standard. ERP-CCK, ERP-DSSS, ERP-OFDM, ERP-PBCC and DSSS-OFDM are all key techniques used in the physical layer for the compatibility for Std. 802.11g [1]. And also carrier sense multiple access with collision avoidance (CSMA/CA) is used as key techniques in WLAN Medium Access Control (MAC) layer, supporting data rates from 1 to $54 \mathrm{Mbps}$.

IEEE $802.11 \mathrm{~g}$ network occupies a bandwidth of about $20 \mathrm{MHz}$ and the available channels are defined with $5 \mathrm{MHz}$ separation between consecutive carriers. As a result, there are only three non-overlapping channels (such as channels $1,6,11$ ) in $2.4 \mathrm{GHz}$. Bearing in mind a scenario with high density of APs, three non-overlapping channels are not enough to guarantee an innocuous coexistence between different WLAN networks. In this circumstance, how to reduce even avoid interference is referred as an important question to network operators. Many studies have presented the analytical study on this category about IEEE 802.11 networks. Co-channel and adjacent channel interference, which are caused by transmissions in entirely or partially overlapping channels, are learned much more recently. The authors in [2] learned the adjacent channel interference in IEEE $802.11 \mathrm{a} / \mathrm{b} / \mathrm{g}$ WLANs and present new analytical and simulation results for the conversational speech capacity of WLANs and compares the different WLAN technologies in that regard. Reference [3] and [4] had done the similar studies focusing on DSSS 
technology about $802.11 \mathrm{~b}$ network and found out some mechanisms may be available for radio resource management in WLANs network, such as transmitted power assignments or rate adaptation strategies. The impact of co-channel interference has been studied in [5] and [6] and the approaches like power adjustment algorithm, channel adjustment algorithm have been proposed for minimizing co-channel interference. In this paper, the author gives study illustrating the performance of one AP characterized by IEEE 802.11g standard, discusses the influence of interference caused by CSMA/CA media access method and channel overlap, especially shows all results in the upper both by simulation and real test method. Then some details are referred to give proposals to network operators how to design a WLAN network more standardized and orderly.

The rest of this document is structured as follows: In Section 2, we give the performance learn of one AP including its coverage performance, load-carrying properties and fairness. Section 3 quantifies the co-channel, adjacent channel interference which should be paid attention to in deploying network services. And a particular introduction to $802.11 \mathrm{~g}$ performance is provided through simulation and test methods. At the end, some proposals are mentioned to network operators and conclude our study.

\section{ONE AP's PERFormanCE}

\subsection{Coverage Performance}

Wide coverage of wireless signal is one consideration of WLAN network deployment. The follow formula is normally used to obtain coverage when doing network planning.

$$
P_{r}=P_{t}-L_{d}+G_{r}+G_{t}-L_{s}
$$

In formula $1, \mathrm{P}_{\mathrm{r}}$ and $\mathrm{P}_{\mathrm{t}}$ are the receiver power and the transmitting power, $\mathrm{L}_{\mathrm{d}}$ and $\mathrm{L}_{\mathrm{s}}$ are the path loss and feeder loss respectively, $G_{r}$ and $G_{t}$ are the gains of the receiver antenna and the transmitting antenna. For calculating the maximum coverage radius of one AP, a threshold of the lowest received power is supposed as $-75 \mathrm{dBm}$ in more than $95 \%$ area. EIRP (equivalent isotropic radiated power) which represents the total effective transmit power of the radio, including gains that the antenna provides and losses from the antenna cable is often used to describe the power limitations for wireless LANs. In the U.S., the FCC defines the transmitting power limitations for wireless LANs in FCC Part 15.247. The FCC declares the EIRP to be 1 watt (1,000 milliwatts) or less when using omni-directional antennas having less than $6 \mathrm{~dB}$ gain in a typical indoor scenario for $2.4 \mathrm{GHz}$ ISM bands. Here, we use the EIRP 100 milliwatts for a typical indoor scenario and 500 milliwatts for a typical outdoor scenario in China for WLAN devices. ITU-R Std. P.1238-7 and Std. P.1411-5 generally represent how to calculate the path loss from $30 \mathrm{MHz}$ to $100 \mathrm{GHz}$ bands of indoor scenario and outdoor scenario separately. However, the parameters about $2.4 \mathrm{GHz}$ ISM bands are not so fit for WLAN network planning in practice. Then we choose the following model referred in [7] and [8] as the path loss model:

$$
\begin{gathered}
\mathrm{L}(\mathrm{d})= \begin{cases}\operatorname{LFS}(\mathrm{d}) & \mathrm{d}<=\mathrm{d}_{\mathrm{BP}} \\
\operatorname{LFS}\left(\mathrm{d}_{\mathrm{BP}}\right)+35 \log 10\left(\mathrm{~d} / \mathrm{d}_{\mathrm{BP}}\right) & \mathrm{d}>\mathrm{d}_{\mathrm{BP}}\end{cases} \\
\operatorname{LFS}(\mathrm{d})=20 * \log (\mathrm{d})+20 * \log (\mathrm{f})-27.6
\end{gathered}
$$


The path loss can be worked out with formula 2 and 3 where $d$ is the distance with the units coming out in meter, $\mathrm{d}_{\mathrm{BP}}$ is the distance of breakpoint with the units being meter and $f$ is the frequency with the units of megahertz. Moreover shadow fading should be considered as:

$$
p(x)=\frac{1}{\sqrt{2 \pi} \sigma} \exp ^{\left(-\frac{x^{2}}{2 \sigma^{2}}\right)}
$$

The authors in [7] and [8] introduced six scenarios including Flat fading (no multipath), Residential, Residential / Small Office, Typical Office, Large Office and Large Space (indoors / outdoors).
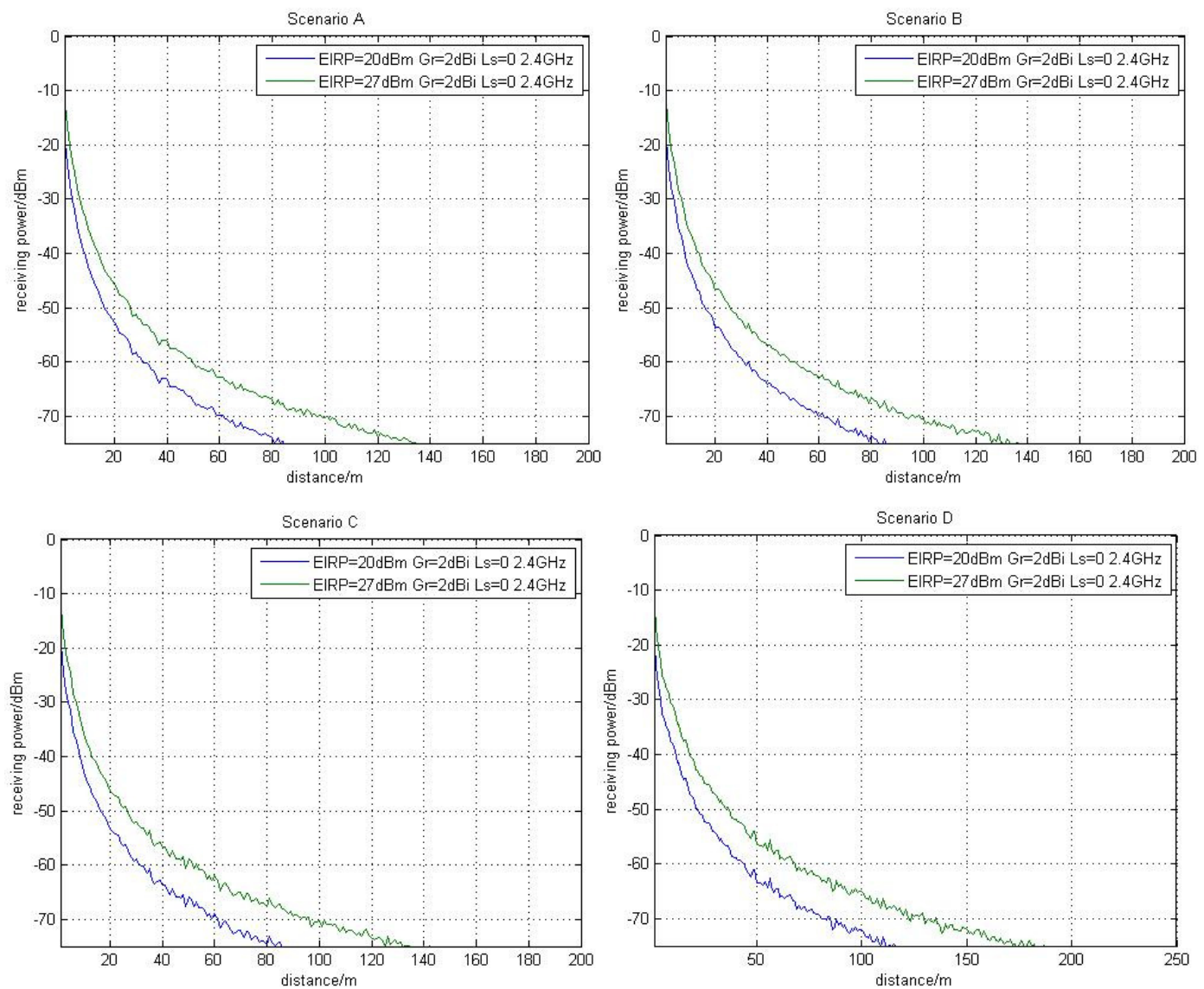

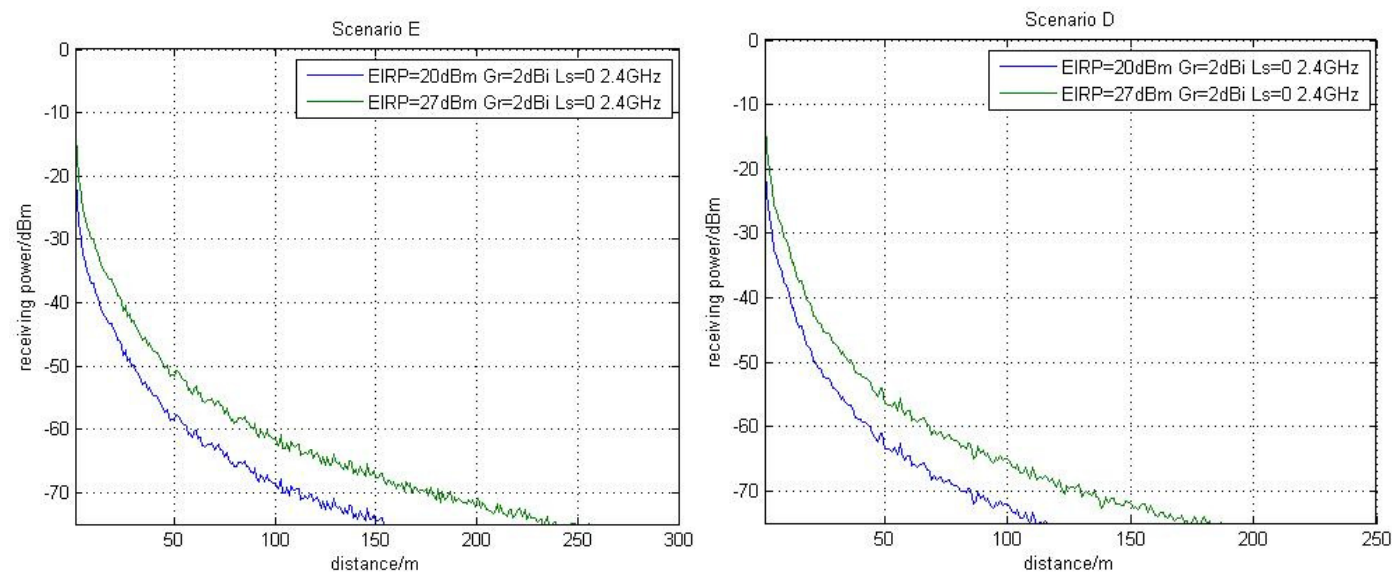

Figure 1. AP's maximum coverage radius under different scenarios

Figure 1 shows one AP's maximum coverage radius under the upper scenarios. From figure 1, we can get that one AP's maximum coverage radius is about $140 \mathrm{~m}$ for $\mathrm{A} \backslash \mathrm{B} \backslash \mathrm{C}$ scenarios and $300 \mathrm{~m}$ for $\mathrm{DLELF}$ scenarios in $2.4 \mathrm{GHz}$ bands. One AP's maximum coverage radius can be manifested in the following table 1 including with the scenarios.

Table 1. One AP's maximum coverage radius

\begin{tabular}{|c|c|c|c|}
\hline Type & Scenarios description & $\begin{array}{c}2.4 \mathrm{GHz}, \\
\mathrm{G}<10 \mathrm{dBi}\end{array}$ & $\begin{array}{c}2.4 \mathrm{GHz}, \\
\mathrm{G}>10 \mathrm{dBi}\end{array}$ \\
\hline A & Flat fading (no multipath) & $90 \mathrm{~m}$ & $140 \mathrm{~m}$ \\
\hline B & Residential & $90 \mathrm{~m}$ & $140 \mathrm{~m}$ \\
\hline C & Residential/Small Office & $90 \mathrm{~m}$ & $140 \mathrm{~m}$ \\
\hline D & Typical Office & $120 \mathrm{~m}$ & $180 \mathrm{~m}$ \\
\hline E & Large Office & $160 \mathrm{~m}$ & $250 \mathrm{~m}$ \\
\hline F & Large Space (indoors/outdoors) & $200 \mathrm{~m}$ & $300 \mathrm{~m}$ \\
\hline
\end{tabular}

\subsection{DCF Fairness}

As we know, DCF is the forcing term in IEEE Std. 802.11. G. Bianchi, in the article [9], modelled the DCF competing process as two-dimensional discrete time Markov chain. Making use of this thesis, the authors intend to prove the fairness of DCF at first.

Lemma 1: Assuming that any $i$ th UE and $j$ th UE have the similar wireless channel condition to access one AP, the $i$ th UE and $j$ th UE will have the same chance to transmit a packet in the current time slot under DCF mechanism.

Proof: Gilles Berger-Sabbatel et.al had been modeled the fairness problem of DCF as Slotted ALOHA [10]. Given a network with one AP and N UEs and $\gamma_{i}$ be the fraction of transmissions performed by UE $i$ during a time slot $m$, the fairness index is the following:

$$
F_{J}(m)=\frac{\left(\sum_{i=1}^{N} \gamma_{i}\right)^{2}}{N \sum_{i=1}^{N} \gamma_{i}^{2}}
$$


Here, we assume that any ith UE and the jth UE have the similar wireless condition to access AP. So we get $\left(\sum_{i=1}^{N} \gamma_{i}\right)^{2}=N \sum_{i=1}^{N} \gamma_{i}^{2}, i=1 \ldots N$, which means $F_{J}(m)=1$. That is to say that any UE with the same wireless condition in the network has the same transmitting probability to access the AP.

Table 2. Parameters setup in fairness simulation

\begin{tabular}{|l|l|l|l|l|}
\hline Parameters & BSS Number & AP Number & UE Number & service \\
\hline Values & 1 & 40 & 10 & FTP \\
\hline Parameters & $\begin{array}{l}\text { DATA } \\
\text { Length }\end{array}$ & Time & Power & $\begin{array}{l}\text { Access } \\
\text { Mechanism }\end{array}$ \\
\hline Values & $1 \mathrm{MB}$ & $0.32 \mathrm{~s}$ & $100 \mathrm{~mW}$ & CTS_self \\
\hline
\end{tabular}

It is turn to prove fairness of DCF by simulation mentioned in the upper case. Announcing a wireless network scene, forty users are located the same distance far away the centre AP at downloading package of $1 \mathrm{M}$ bytes in every $0.32 \mathrm{~s}$. The users' transmitting speed is 54Mbps and CTS_self protocol are used in MAC layer. All parameters setup in this simulation are listed in table 2. Without other limitation, we can get forty users' total throughput is about $20.8 \mathrm{Mbps}$ and every user is about $0.5 \mathrm{Mbps}$ from figure 2 . From this simulation result, we can get forty users have the same chance to access the channel and transmit packets. Thus, it is unfitted for network operators to let all users compete with each other completely because CTS_self protocol can bring out the possibility of collision severely which will lead to the decrease of total throughput and single user's throughput.

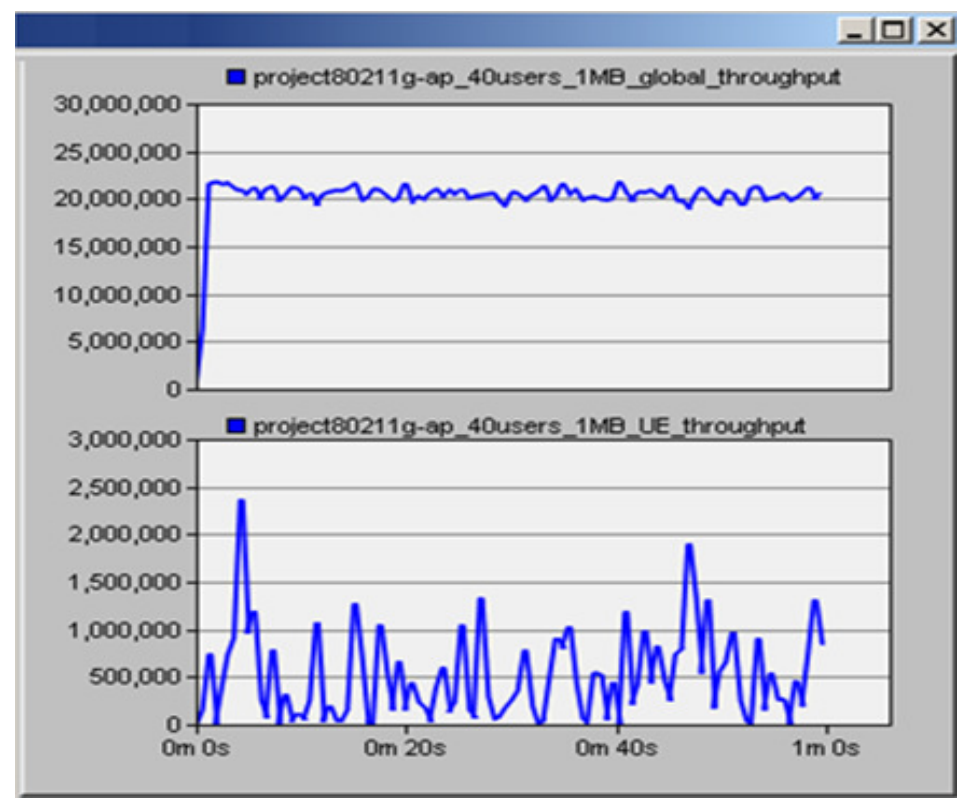

Figure 2. 40 UEs total throughput and 1UE's under DCF mechanisms 
International Journal of Wireless \& Mobile Networks (IJWMN) Vol. 5, No. 5, October 2013

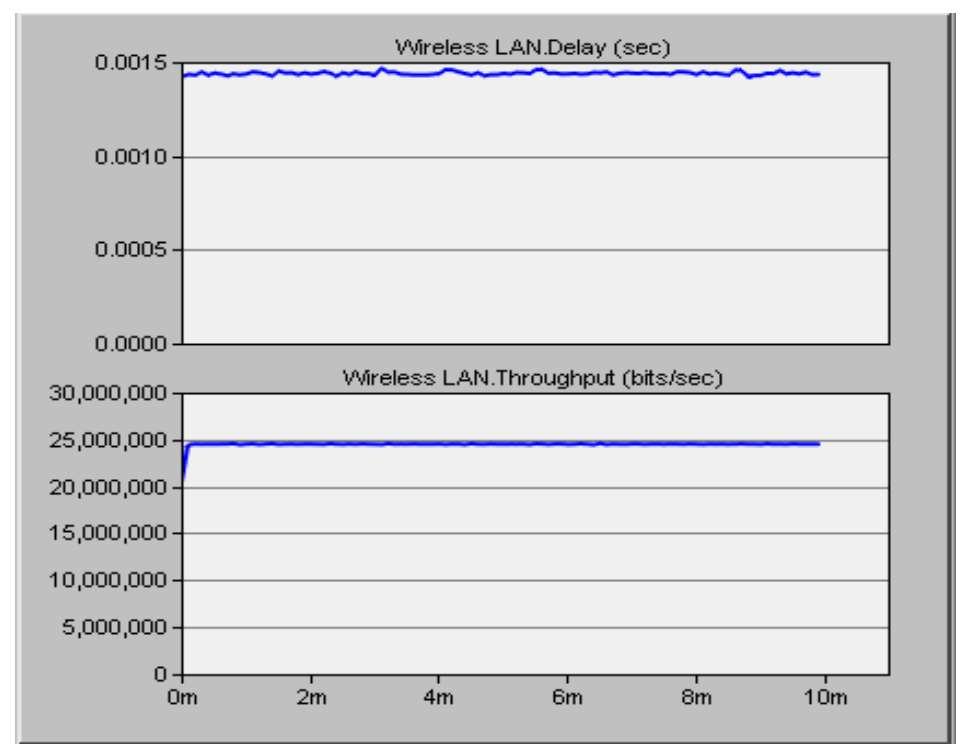

Figure 3. One UE's throughput and time delay

\subsection{Load-carrying properties with TCP Protocol}

Many articles had learned the performance of 802.11 wireless network's by theoretical analysis like [9], [10] etc. We check the performance by simulation and test methods. First, we give out a simulation to show the wireless network's performance of application layer with TCP protocol. Supposing that a BSS contains $1 \mathrm{UE}$ only, this user is downloading package of $1 \mathrm{M}$ bytes in every 0.32s from the centre AP. The users' transmitting speed is 54Mbps and CTS_self protocol are used in MAC layer. That is to say that we use the parameters shown in table 2 but the UE Number is 1 in this simulation. From figure 3, this user's throughput is about 24.5Mbps and the time delay is less than $2 \mathrm{~ms}$. Compared figure 2 with figure 3 , we can get complete competition can lead to the 40 users' total throughput decreasing approximated $4.1 \mathrm{Mbps}$.

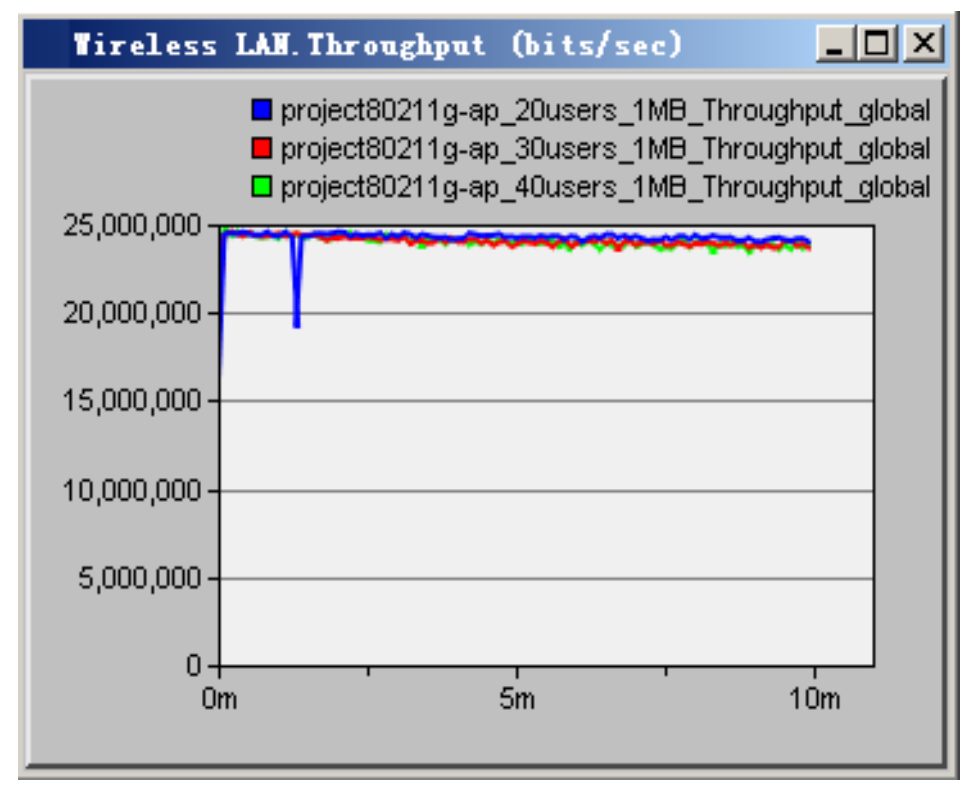

Figure 4. Multiple UEs total throughput with optimal configuration 


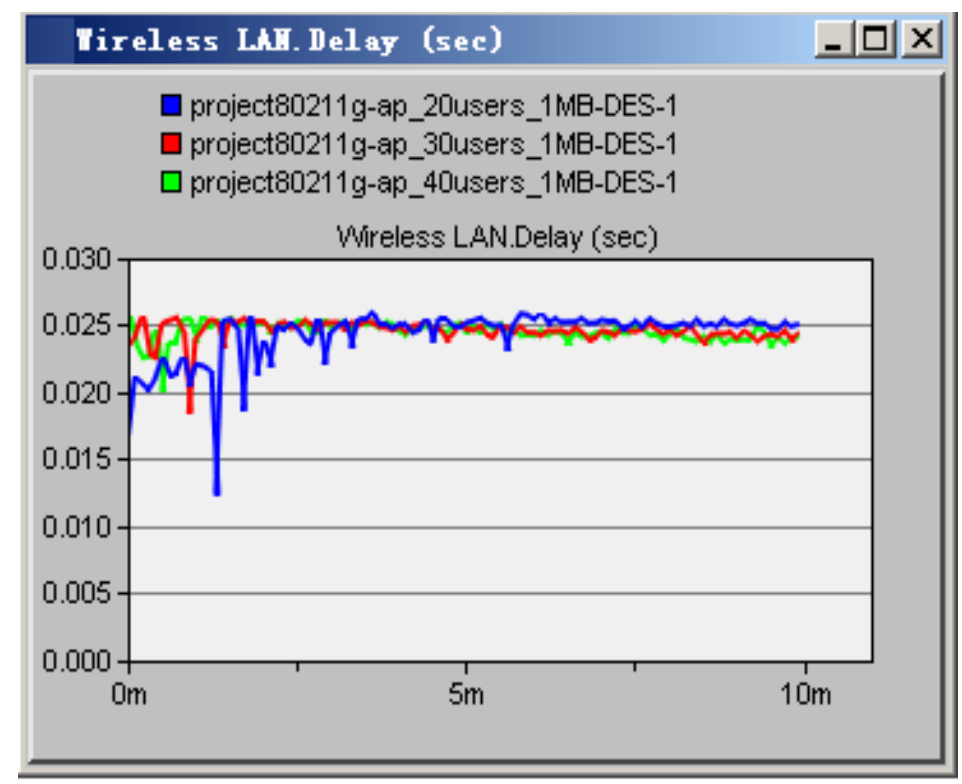

Figure 5. Multiple UEs time delay with optimal configuration

Here, a question is coming into notice. Whether can the collision be avoided or reduced under CTS_self protocol? In the following, supposing that a BSS contains $\mathrm{N}=20,30,40$ users respectively and all users are downloading package of $1 \mathrm{MB}$ with the transmitting speed 24.5/ $N$ Mbps (other parameters are like in table 2), we optimally configure the network parameters and use CTS_self protocol by the MAC layer accessing. Figure 4 depicts the total throughput of the network and figure 5 recounts the time delay of every circumstance. It is observed that the total throughput of the network is about $24.5 \mathrm{Mbps}$ and the time delay is increasing when the network becomes a dense one. That is to say, optimal configuration can make the collision be avoided or reduced, improving the WLAN network's carrying capacity and making it more efficiently.

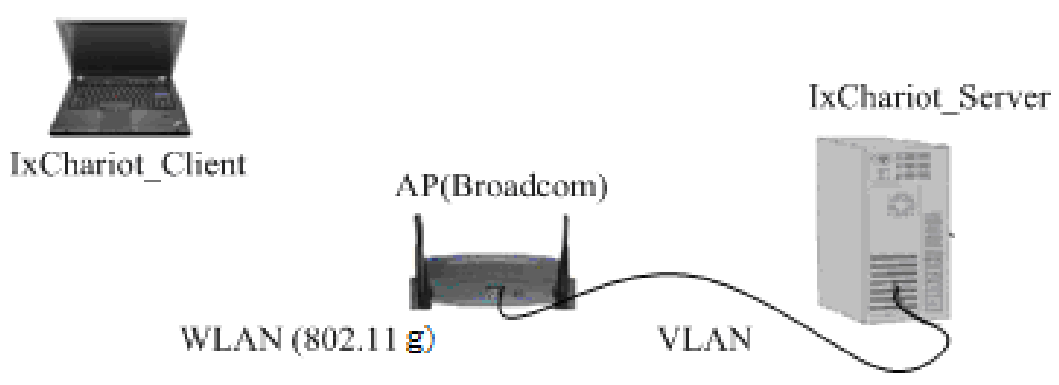

Figure 6. A test scenario for one BSS's performance

To improve the upper situation results, we give out a test for one BSS's throughput, a small scale 802.11g network was set up in the office environment like figure 6. Ixchariot_Client is the ftp client fixed on PC, Ixchariot_Server is the ftp server fixed on server, AP is connected with sever by VLAN, and PC is connected with AP by 802.11g WLAN network. In this case, network coverage is out of our consideration, so free-space attenuation is supposed for the test thus the loss is a little larger indeed. 
International Journal of Wireless \& Mobile Networks (IJWMN) Vol. 5, No. 5, October 2013

Table 3. Parameters configuration in the test of the BSS's Throughput

\begin{tabular}{|l|l|l|l|l|}
\hline Parameters & $\begin{array}{l}\text { BSS } \\
\text { Number }\end{array}$ & $\begin{array}{l}\text { AP } \\
\text { Number }\end{array}$ & $\begin{array}{l}\text { UE } \\
\text { Number }\end{array}$ & service \\
\hline Values & 1 & 1 & 1 & FTP \\
\hline Parameters & $\begin{array}{l}\text { DATA } \\
\text { Length }\end{array}$ & Time & Power & $\begin{array}{l}\text { Access } \\
\text { Mechanism }\end{array}$ \\
\hline Values & 1MB & $0.32 \mathrm{~s}$ & $100 \mathrm{~mW}$ & CTS_self \\
\hline
\end{tabular}

At first, we let one Ixchariot_Client link the AP by downloading the package of $1 \mathrm{MB}$ bytes in MAC layer and other network configuration looks like the table 3.The largest throughput of the BSS can be gotten in figure 7. That is to say the maximal throughput of application layer is nearly 22.5 Mbps, a little less than simulation result. Then we ping the AP from the UE simultaneously, we can obtain the total time delay of network is nearly $3 \mathrm{~ms}$ in figure 8 . The test results are little worse than the simulation scene because other attenuation maybe occur during the real environment. Figure 7 and figure 8 confirm that our simulation results are correct basically.

FTP DOWNLOAD

Hot spot:Broadcom [00: 90:4c: 09: 24: c4]

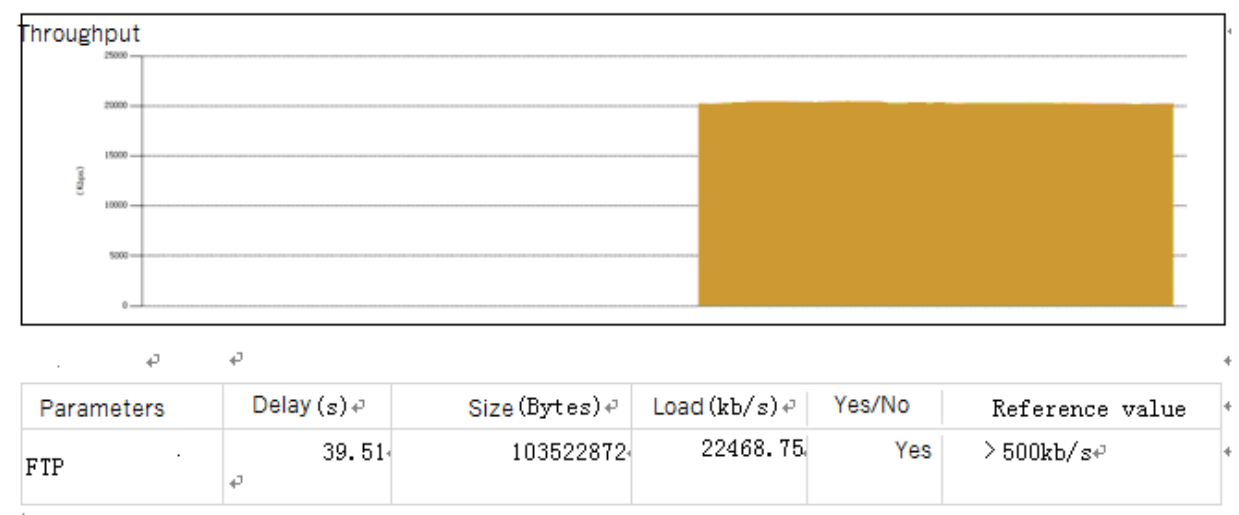

Figure 7. One BSS's throughput with FTP protocol

Ping Time Delay

Hot spot: Broadcom [00:90:4c: 13:24:c4]

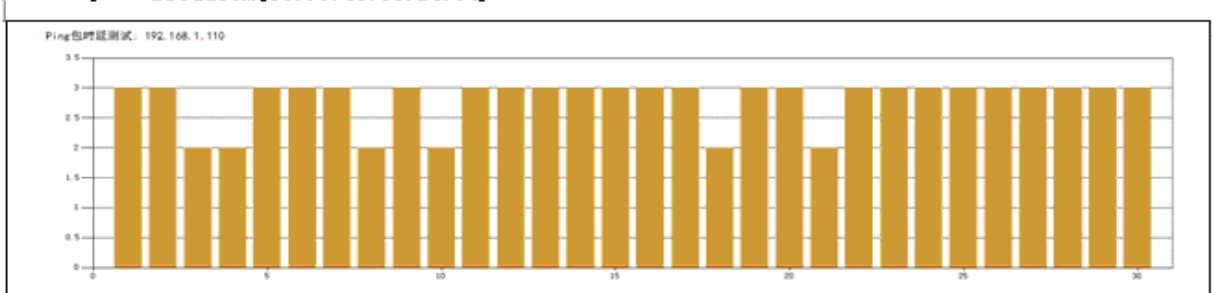

\begin{tabular}{|c|c|c|c|c|c|}
\hline Parameters & Ping Count & Yes times & No times & Success rate & Reference value \\
\hline Ping rate & 30 & 30 & 0 & $100 \%$ & $>95 \%$ \\
\hline Parameters & Ping Count & Delay(Max) & Delay(Min) & Delay(AVG) & Reference value \\
\hline Ping delay & 30 & $3 \mathrm{~ms}$ & $2 \mathrm{~ms}$ & $2 \mathrm{~ms}$ & $<200 \mathrm{~ms}$ \\
\hline
\end{tabular}

Figure 8. Ping time delay of the network with FTP protocol 


\section{THE CONTRAST BETWEEN CO-CHANNEL INTERFERENCE AND ADJACENT CHANNEL INTERFERENCE}

As we know, 802.11g uses OFDM modulation to support the data rate 54Mbps. OFDM system is easily affected by the frequency deviation, which makes the subcarrier orthogonality be destroyed, resulting in mutual interference between signals of different channels. To evaluate the interference, we suppose a scenario with two WLAN networks like figure 9. Presumption is made as every 20 UEs accessing the same channel in one BSS and assuming every 20 UEs randomly located in one cell. Access Point_1, server 1 and Mobile_1_x (x is instead of the subscript from 1 to 20) compose the basic structures of BSS1 and other instruments are e basic structures ofBSS2 respectively. APPL icons are the application definition and application profile defined for the service for two BSSs.

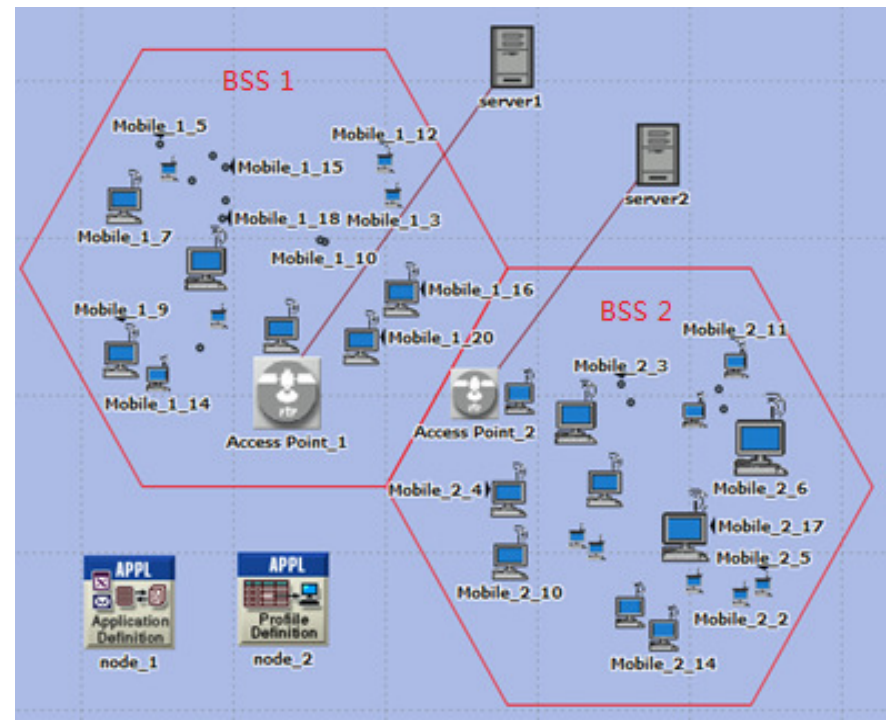

Figure 9. Interference simulation scene of two BSS

Presumption is made as every 20 UEs accessing the same channel in one BSS and assuming every 20 UEs randomly located in one cell. The users' transmitting speed is 54Mbps and CTS_self protocol are used in MAC layer. The free space model is used to calculate path loss, the users' transmitting power is $100 \mathrm{~mW}$ and all UEs and Aps both in BSS1 and BSS2 are set to use channel 1/ channel 1, channel 1/ channel 2, channel 1/ channel 3, channel $1 /$ channel 4 and channel 1/ channel 6 to communication separately. All parameters set for two BSSs are listed in Table 4.

Table 4. Parameters configuration in the test of the BSS's Throughput

\begin{tabular}{|l|l|l|l|l|l|}
\hline $\begin{array}{l}\text { Parameters } \\
\text { of BSS 1 }\end{array}$ & $\begin{array}{l}\text { BSS } \\
\text { Number }\end{array}$ & $\begin{array}{l}\text { AP } \\
\text { Number }\end{array}$ & $\begin{array}{l}\text { UE } \\
\text { Number }\end{array}$ & service & $\begin{array}{l}\text { Channel } \\
\text { Number }\end{array}$ \\
\hline $\begin{array}{l}\text { Values of } \\
\text { BSS 1 }\end{array}$ & 1 & 1 & 20 & FTP & 1 \\
\hline $\begin{array}{l}\text { Parameters } \\
\text { of BSS 1 }\end{array}$ & $\begin{array}{l}\text { DATA } \\
\text { Length }\end{array}$ & Time & Power & $\begin{array}{l}\text { Access } \\
\text { Mechanism }\end{array}$ & Rate \\
\hline Values of & $1 \mathrm{MB}$ & $0.82 \mathrm{~s}$ & $100 \mathrm{~mW}$ & CTS_self & 54Mbps \\
\hline
\end{tabular}


International Journal of Wireless \& Mobile Networks (IJWMN) Vol. 5, No. 5, October 2013

\begin{tabular}{|l|l|l|l|l|l|}
\hline BSS 1 & & & & & \\
\hline $\begin{array}{l}\text { Parameters } \\
\text { of BSS 2 }\end{array}$ & $\begin{array}{l}\text { BSS } \\
\text { Number }\end{array}$ & $\begin{array}{l}\text { AP } \\
\text { Number }\end{array}$ & $\begin{array}{l}\text { UE } \\
\text { Number }\end{array}$ & service & $\begin{array}{l}\text { Channel } \\
\text { Number }\end{array}$ \\
\hline $\begin{array}{l}\text { Values of } \\
\text { BSS 2 }\end{array}$ & 2 & 1 & 20 & FTP & $1 / 2 / 3 / 4 / 6$ \\
\hline $\begin{array}{l}\text { Parameters } \\
\text { of BSS 2 }\end{array}$ & $\begin{array}{l}\text { DATA } \\
\text { Length }\end{array}$ & Time & Power & $\begin{array}{l}\text { Access } \\
\text { Mechanism }\end{array}$ & Rate \\
\hline $\begin{array}{l}\text { Values of } \\
\text { BSS 2 }\end{array}$ & $1 \mathrm{MB}$ & $0.82 \mathrm{~s}$ & $100 \mathrm{~mW}$ & CTS_self & 54Mbps \\
\hline
\end{tabular}

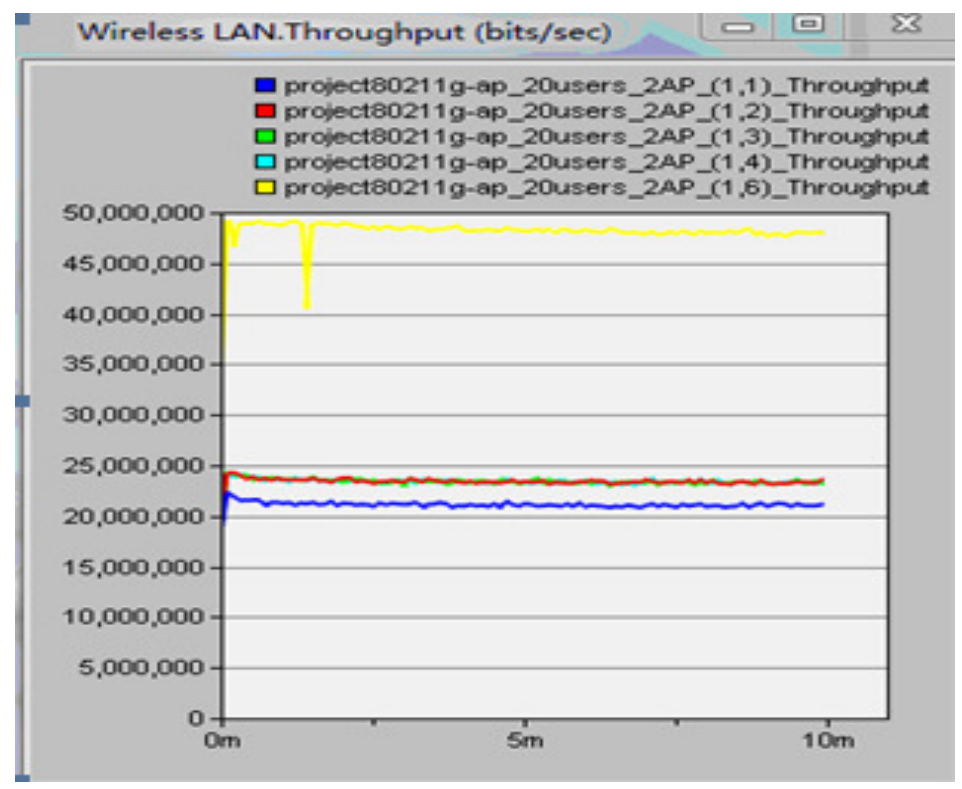

Figure 10. (a) the total throughput contrast

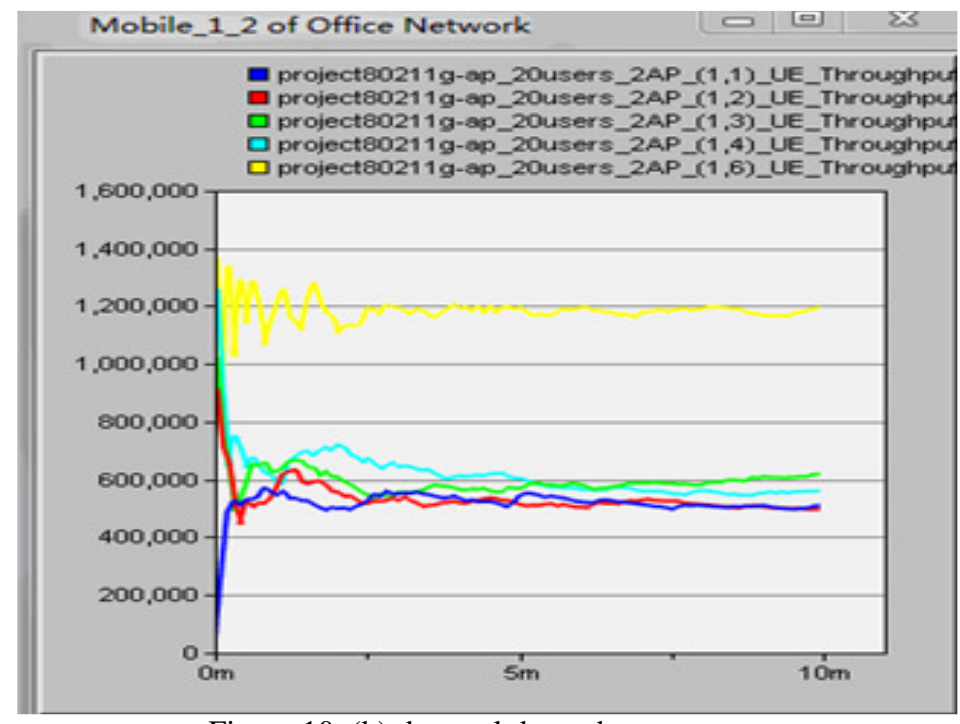

Figure 10. (b) the total throughput contrast 
The lines in figure 10 are named project name with project 802.11g _AP, 20 users in one BSS, 2 APs' contrast, channel numbers of two BSSs, and total throughput or throughput of single UE. The throughput of different channel model is distinguished by different colours. When all users are downloading package of $1 \mathrm{M}$ bytes in every $0.82 \mathrm{~s}$, we can get the total throughput is about 21.6, 24, 24, 24 and 49Mbps of the network separately from figure 10 (a). And also, in figure 10 (b), the throughput is about $500,500,580,600$ and $1200 \mathrm{~K}$ bytes of one UE, which shows the interference is serious when the channel is overlapped.

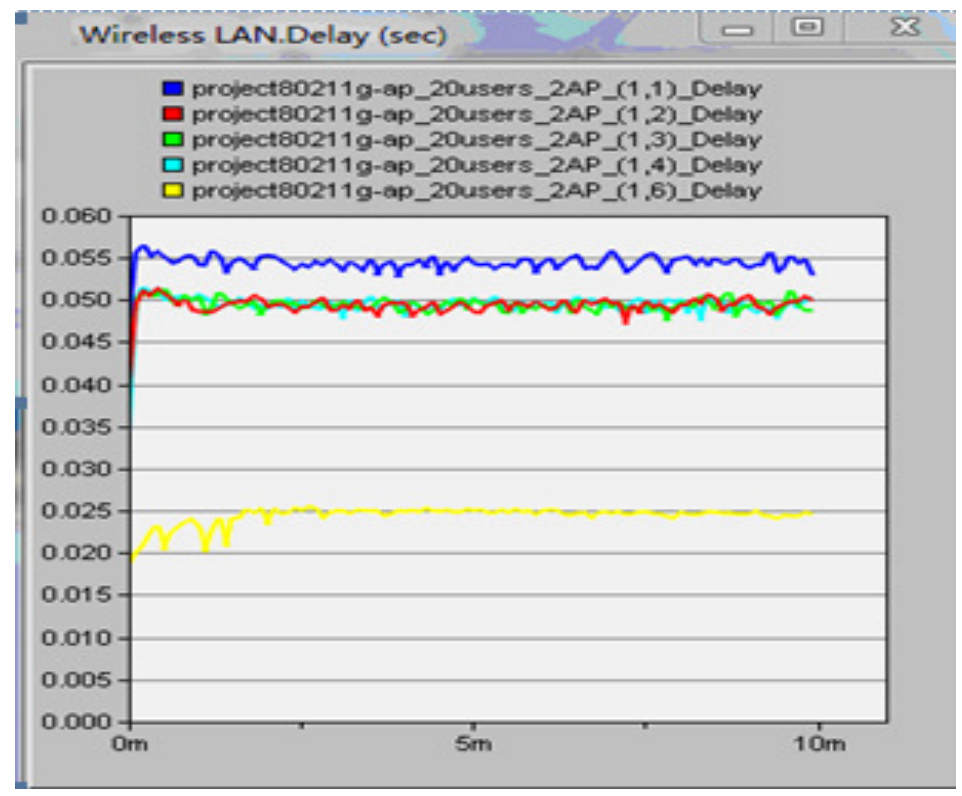

Figure 11. (a) the total time delay contrast

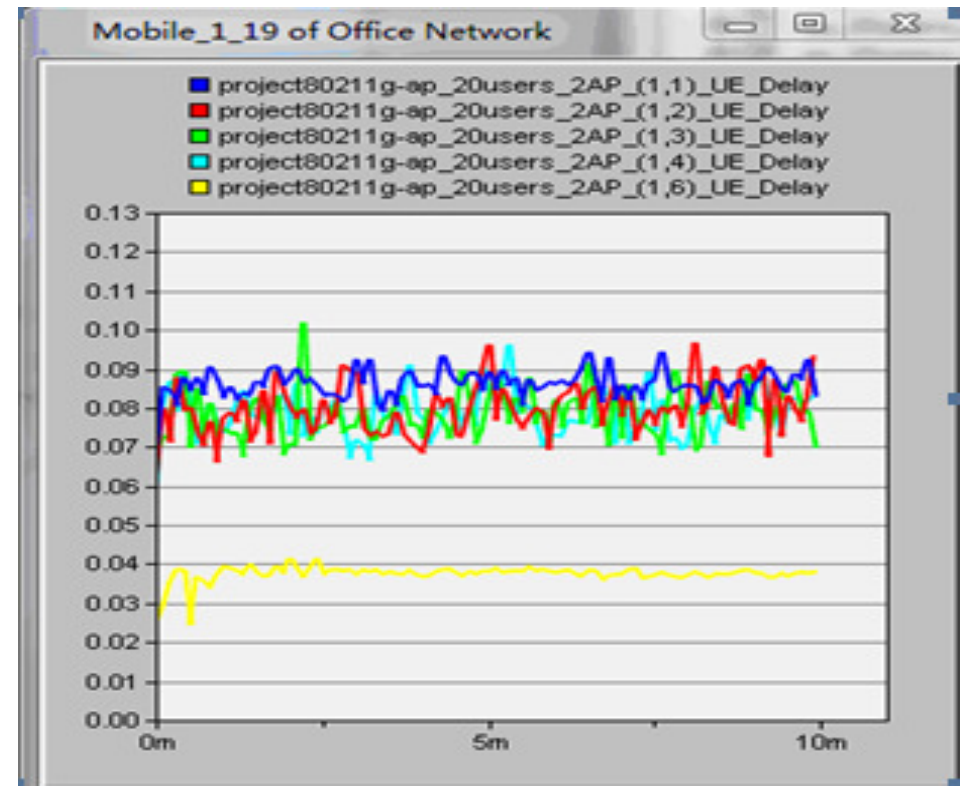

Figure 11. (b) the time delay comparison of one UE 
Figure 11 is named like figure 10, and also, the different channel model is distinguished by different colours. Figure 11 (a) and (b) show the total time delay of the network and the time delay of one UE. It also confirms that additional RF medium contention overhead occurs for all radios using the same channel in physical area resulting in throughput degradation and latency. With the upper comparison, we can infer that $2.4 \mathrm{GHz}$ ISM bands cannot support more than three Aps work independently simultaneously. Actually, the authors find out the collision cannot be avoided if the interference cannot be limited tiny enough when the channel is detected based on energy detection.

\section{CONCLUSION}

In this paper we have proposed much analysis, simulation case and real test to illustrate the performance of $802.11 \mathrm{~g}$ application layer and the fairness issues related to DCF, even analyze the co-channel, adjacent channel interference. Let us recall here that our final goal is to suggest the details which should be paid attention to in deploying network services. First, one AP's coverage performance, load-carrying properties and fairness should be considered, with the constraint of DCF, the largest user number is less the 40 in a BSS (Actually, 20 users is much better in a BSS within $802.11 \mathrm{~g}$ WLAN network).Second, for minimizing the Co-channel Interference and adjacent channel interference, WLAN network operator have to find other method to achieve loading balance for users with different channel condition in real circumstance. And the last one is that the simulation results and test results are general values which can be offered as the basis.

\section{ACKNOWLEDGEMENTS}

This work is supported by Important National Science and Technology Specific Projects NO.2013ZX03003016.

\section{REFERENCES}

[1] IEEE Computer Society, (2003) "Wireless LAN Medium Access Control (MAC) and Physical Layer (PHY) Specifications," IEEE Std.802.11-2003.

[2] K. Medepalli, et al., "Voice capacity of IEEE 802.11b, 802.11a, and 802.11g wireless. LANs," Proceedings of the IEEE Globecom, 2004, pp. 1549-1553.

[3] Eduard Garcia Villegas,Elena Lopez-Aguilera, Rafael Vidal and Josep Paradells, (2007) "Effect of adjacent-channel interference in IEEE 802.11 WLANs," 2nd International Conference on Cognitive Radio Oriented Wireless Networks and Communications, pp.118-125.

[4] Bicket, J., Aguayo, D., Biswas, S., and Morris, R., (2005) "Architecture and Evaluation of an Unplanned 802.11b Mesh Network," Proceedings of the 11th annual international conference on Mobile computing and networking, pp. 31-42.

[5] Broustis I., PapagiannakiK., Krishnamurthy S.V., et.al., (2009) "Measurement-Driven Guidelines for 802.11 WLAN Design ,” IEEE/ACM Transactions on Networking, Vol 18, No.3, pp.722-735.

[6] H. Wu, Y. Peng, K. Long, J. Ma, (2002) "Performance of Reliable Transport Protocol over IEEE 802.11 Wireless LAN: Analysis and Enhancement,” Proceedings of IEEE INFOCOM, vol.2, pp. 599607.

[7] Erceg, V., Schumacher, L., Kyritsi, P., et.al., (2004) “TGn Channel Models,” IEEE 802.11-03/940r4.

[8] Stephens, A., Bjerke, B., Jechoux, B., et.al., (2004) “Usage Models, ” IEEE 802.11-03/802r23.

[9] G. Bianchi,(2000) "Performance analysis of IEEE 802.11 distributed coordination function," IEEE J. Select. Areas. Commun., vol. 18, no. 3, pp.535-547. 
International Journal of Wireless \& Mobile Networks (IJWMN) Vol. 5, No. 5, October 2013

[10] E. Lopez, J. Casademont, J. Cotrina , (2004) "Outdoor IEEE 802.11g Cellular Network performance," Proceedings of IEEE Globecom04, Vol.5, pp.2992-2996.

\section{Authors}

Mingming Li works in State Radio Monitoring Center of China as an engineer. Her research field is frequency spectrum planning and spectrum demand forecasting. 